\title{
Selective tuning of the right inferior frontal gyrus during target detection
}

\author{
Adam Hampshire, Russell Thompson, John Duncan, And Adrian M. OWen \\ Cambridge University, Cambridge, England
}

\begin{abstract}
In the human brain, a network of frontal and parietal regions is commonly recruited during tasks that demand the deliberate, focused control of thought and action. Previously, using a simple target detection task, we reported striking differences in the selectivity of the BOLD response in anatomically distinct subregions of this network. In particular, it was observed that the right inferior frontal gyrus (IFG) followed a tightly tuned function, selectively responding only to the current target object. Here, we examine this functional specialization further, using adapted versions of our original task. Our results demonstrate that the response of the right IFG to targets is a strong and replicable phenomenon. It occurs under increased attentional load, when targets and distractors are equally frequent, and when controlling for inhibitory processes. These findings support the hypothesis that the right IFG responds selectively to those items that are of the most relevance to the currently intended task schema.
\end{abstract}

In a recent article, we used event-related fMRI to compare the neural responses in distinct subregions of the frontal and parietal cortices during a simple target detection task (Hampshire, Duncan, \& Owen, 2007). Participants were initially presented with a target object: a face, building, abstract shape, or line figure. Subsequently, they monitored sequences of distractor objects for occasional presentation of the target. We reported striking differences in selectivity of the BOLD response in different frontoparietal regions. Whereas the more ventral and posterior extent of the lateral prefrontal cortex (inferior frontal gyrus, or IFG) responded selectively only to the current target object, more dorsal and anterior portions (middle frontal gyrus, or MFG) and the inferior parietal cortex (IPC) were more active during the presentation of both targets and distractors from the same category as the target. A lateralization effect was also evident, favoring heightened BOLD response to targets in the right hemisphere. Our results suggested that within the frontoparietal cortex, the right IFG responds particularly selectively when the attended input matches the current focus of intended action (Owen \& Hampshire, in press).

More broadly, evidence from a range of sources has suggested that there is an adaptable global system for working memory and attention, distributed across a network of frontal and parietal brain regions. This global attentional network appears to underlie the flexibility of human behavior by enabling us to deliberately and selectively focus our attention on currently relevant information (Duncan, 2001, 2006; Miller \& Cohen, 2001). In support of this hypothesis, it has been reported that, within the human brain, a consistent set of frontal and parietal regions is com- monly recruited under a broad range of cognitive demands (Braver, Reynolds, \& Donaldson, 2003; D'Esposito, Postle, Ballard, \& Lease, 1999; Dove, Pollmann, Schubert, Wiggins, \& von Cramon, 2000; Dreher, Koechlin, Ali, \& Grafman, 2002; Hampshire \& Owen, 2006; Kimberg, Aguirre, \& D'Esposito, 2000; Sohn, Ursu, Anderson, Stenger, \& Carter, 2000). Research using single unit recording also supports this hypothesis, with neurons within the monkey lateral prefrontal cortex rapidly adapting to respond to a broad range of task-relevant information yet becoming unresponsive to that same information when the task demands change (Everling, Tinsley, Gaffan, \& Duncan, 2002; Freedman, Riesenhuber, Poggio, \& Miller, 2001; Miller \& Cohen, 2001).

Although the existence of a frontoparietal network for coding of task-relevant information is now widely accepted, the contributions made by the anatomically distinct subregions of this network remain controversial. Of particular relevance to the present study are several influential models that propose that the lateral prefrontal cortex is functionally organized along an inferiorsuperior (or ventral-dorsal) axis. Prominent among these models are the suggestions that the inferior and superior regions of the lateral prefrontal cortex are differentially involved in first order versus higher order executive functions (Petrides, 1994, 1995, 2005), the active maintenance versus the controlled manipulation of items in working memory (D'Esposito et al., 1999; Owen, 1997), and the maintenance versus the structuring of working memory items (Bor, Duncan, \& Owen, 2001). Although these models have in common the suggestion that the IFG and MFG are recruited during different task demands, the un- 
derlying cognitive basis remains unclear. In part, this is due to the use of complex, cognitively heterogeneous task manipulations that tend to confound a number of factors when attempting to functionally dissociate subregions of the frontoparietal network - for example, expectancy, difficulty, and arousal (Fletcher \& Henson, 2001). This difficulty in interpreting the basis of reported frontoparietal dissociations is further exacerbated by the fact that the subregions of this network are just as frequently reported to coactivate (Duncan, 2001, 2006; Duncan \& Owen, 2000; Peelen, Heslenfeld, \& Theeuwes, 2004; Postle \& D'Esposito, 2000). In fact, in some cases, studies manipulating cognitive factors that have previously been reported to dissociate frontal and parietal subregions, but that use subtly different designs, have instead reported coactivation of those same subregions (Peelen et al., 2004; Postle \& D'Esposito, 2000). The observation of a functional dissociation between the IFG and the MFG during the simplest of target detection tasks (Hampshire et al., 2007) therefore adds a valuable insight into the nature of frontoparietal organization; however, replication in a different population sample and under varied task demands is essential to confirm the reliability of this result.

Here, we attempted to refine our understanding of the conditions under which the right IFG is preferentially recruited using a series of modified versions of our original task design. We first sought to replicate our recent finding of an IFG-MFG dissociation, but under increased attentional demand. Participants monitored sequences of compound stimuli that consisted of overlapping faces and buildings for the presentation of a frequently redefined target object (see Figure 1). All faces and buildings were, at different stages, either targets or distractors, and because the stimuli were compound, an object from the same category as that of the current target and an object from the nontarget category were always present. Increased topdown attentional control was required, therefore, in order to resolve the face/building compound pairs.

Classically, target detection paradigms involve monitoring for an infrequently displayed target object (Linden et al., 1999). This imbalance in the target and distractor frequencies is used to ensure that working memory and attention are oriented toward the target object in particular, since it is altogether likely that if a target and distractor occurred equally often, the individual would redirect their intended task schema from detecting the target in particular to sorting between two alternative stimuli of equal relevance to performance. A consequence of this difference in the relative frequencies of targets and distractors is that the increased BOLD response during the presentation of targets could be caused by the orienting of attention to infrequent or surprising events (Corbetta \& Shulman, 2002), as opposed to the recognition of the specific target identity. Here, the first version of our task conformed to the classical design, with targets occurring at a lower frequency than distractors. In order to test whether the selective response of the right IFG to targets was dependent on the relative infrequency of target occurrence, a second version of the task was also undertaken. In this second version of the task, targets and distractors occurred

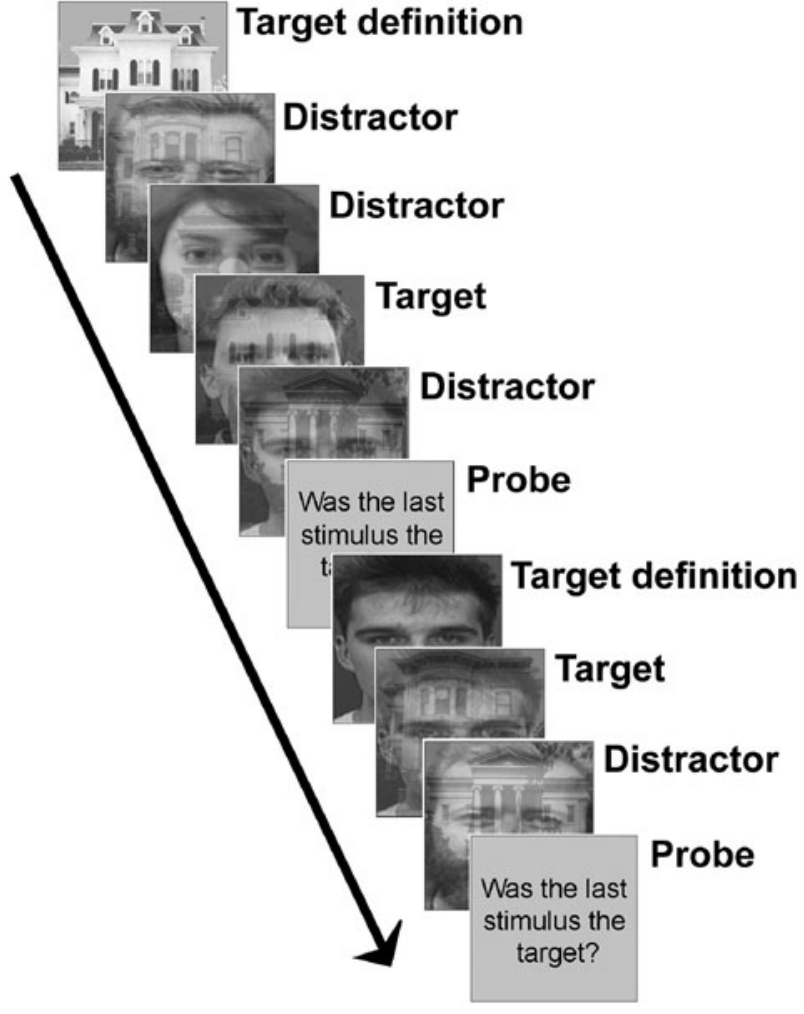

Figure 1. Task design. Volunteers monitored sequences of compound face/building stimuli for the presentation of a target face or building. Overt responses were made only at the time of probes, subsequent to which a new target item was displayed, and a new sequence was monitored.

equally often, and any increase in the BOLD response to targets could not, therefore, be a result of attentional orienting to infrequent or surprising events.

The right IFG has been repeatedly implicated in tasks that involve response inhibition, most notably during go/ no-go tasks, in which a frequent response has to be inhibited on the basis of an infrequently presented cue to stop (Aron, Fletcher, Bullmore, Sahakian, \& Robbins, 2003a, 2003b; Rubia, Smith, Brammer, \& Taylor, 2003). To assess the role of response inhibition, a further cohort undertook two modified versions of the task in which responses were made either (1) only when probed at the end of the sequence as in our original experiment, or (2) whenever an infrequent target was detected, thereby removing any possible requirement for response inhibition. It was our prediction that the heightened BOLD response to targets in the right IFG would be robust across all permutations of the task, regardless of attentional demand, target frequency, and the presence or absence of an immediate motor response.

\section{METHOD}

Tasks 1 and 2: Attentional Load and Target Frequency

Participants were instructed to look for a visually displayed target object within sequences of overlapping face/building stimuli (Figure 1). At the beginning of each sequence, a new target item 
was presented with the word target underneath for 2,000 msec. New target displays consisted of either a single face stimulus or a single building stimulus, and as well as defining particular target stimuli for the upcoming sequence, also implicitly defined the current target category. Once the target stimulus had been defined, presentation of the sequence of compound face/building stimuli began. Each item in the sequence was displayed for $750 \mathrm{msec}$ and was followed by an interstimulus interval (ISI) that lasted from 250 to $750 \mathrm{msec}$. At the end of each sequence a probe stimulus consisting of the question "Was the last stimulus the target?" appeared on the screen for $2,500 \mathrm{msec}$, and participants were required to respond "yes" or "no" with the first two digits of their right hand using a buttonbox. Subsequently, there was an ISI of between $250-750 \mathrm{msec}$ before a new target was displayed and a new sequence began. Although sequences typically contained multiple targets and distractors, participants were explicitly instructed to respond solely on the basis of the stimulus immediately preceding the probe question. Since the sequence length varied unpredictably from 1 to 7 items, however, volunteers were required to attend throughout, mentally classifying each stimulus as the target or a distractor. As responses were required only when the probe appeared at the end of a sequence, we could compare neural activity associated with target and distractor presentation independently from neural activity associated with probes and overt motor responses.

Fourteen right-handed participants between the ages of 20 and 40 undertook the fMRI task. In the first version of the task, data were collected in two blocks of scanning acquisition, each block containing 31 stimulus sequences and lasting for a total of 9 min. Six distinct pictures were used for both stimulus categories, and all pictures were used as both target and distractor items at different stages of the experiment. All stimuli were unfamiliar to participants before they began. Volunteers monitored 250 compound stimuli over the two experimental blocks, 100 of which contained a target object, and the remaining 150 of which contained one of the nontargets from the same category. The sequences were predefined and pseudorandomized to allow target- and distractor-related neural activity to be separately calculated in a rapid event-related design. The presentation of targets and distractors was balanced across the experimental block so that the relative probabilities of each one occurring were equivalent across all seven positions in the stimulus sequence. This design averaged out any effects due to reconfiguration to a new target object, or the expectancy of an impending probe.

The same group of 14 participants also undertook a second version of the task identical to the first, except that the stimulus set was smaller, consisting of just two faces and two buildings. Targets and same-category distractors were, therefore, presented at a $1: 1$ ratio (i.e., $50 \%$ of the compound stimulus pairs contained the target object). This manipulation controlled for the effects of stimulus frequency, target frequency, and the frequency of prepared positive response. Participants undertook two 9-min blocks of scanning acquisition, during each of which they monitored 31 sequences. Participants monitored a total of 250 compound stimulus pairs over the two acquisition blocks, 125 of which contained the target and 125 of which contained a distractor from the same category as the target.

Scanning was carried out at the Wolfson Brain Imaging Centre, Cambridge, using a 3 Tesla Bruker Medspec s300 scanner (Bruker Medspec, Ettingen, Germany). Twenty-one 4-mm slices (1-mm interslice gap) were acquired, using a repetition time (TR) of $1.1 \mathrm{sec}$ and in-plane resolution of $3.125 \times 3.125 \mathrm{~mm}$. A total of $540 \mathrm{~T} 2$ weighted echo-planar images depicting BOLD contrast were acquired per block, with the first 18 discarded to avoid T1 equilibrium effects. The experiment was programmed in Visual Basic 6. Displays were projected onto a screen, visible from the scanner via a mirror, with stimuli subtending a visual angle of $6^{\circ}$.

Images were preprocessed and analyzed using SPM2 (Wellcome Department of Cognitive Neurology). Prior to analysis, images were slice time corrected, reoriented to correct for subject motion, geometrically undistorted using phase maps (Cusack, Brett, \& Osswald, 2003), spatially normalized to the standard Montreal Neurological
Institute template, and smoothed with an 8-mm full-width at halfmaximum Gaussian kernel. Data were also high-pass filtered prior to analysis (cutoff period, $128 \mathrm{sec}$ ). Separate fixed effects analyses were carried out on each volunteer's data for the two versions of the task using general linear models. Regressor functions were created by convolving timing functions indicating the onset and duration of each event with a basis function representing the canonical hemodynamic response. Each regressor function was then entered into the model as a predictor variable. In the present study, each block of trials was modeled by seven predictor variables, each representing one of seven experimental event types: face target definition events, building target definition events, face target presentations (i.e., face target overlapping with ignored building), face distractor presentations (i.e., face distractor overlapping with ignored building), building target presentations (i.e., building target overlapping with ignored face), building distractor presentations (i.e., building distractor overlapping with ignored face), and the probe stimulus with response.

Group level analyses were carried out using six regions of interest (ROIs) from the automated anatomical labeling templates (TzourioMazoyer et al., 2002) representing the IFG (conjunction between the inferior operculum and inferior triangularis), the MFG, and the IPC. For each participant, responses to target and distractor stimuli estimated by the fixed effects analyses were averaged across voxels within each of these ROIs, using the MARSBAR toolbox (Brett, Anton, Valabregue, \& Poline, 2002). These mean values were then exported for analysis using SPSS.

Another analysis was also carried out to examine the effects of attentional modulation on category-specific brain regions. For each volunteer, contrasts were carried out comparing the level of response to face target definition events and to building target definition events. The results of these contrasts were then entered into a random effects analysis and the resulting group-level activation maps examined for regions corresponding to the fusiform face area (FFA) (Kanwisher, McDermott, \& Chun, 1997) and the parahippocampal place area (PPA) (Epstein \& Kanwisher, 1998). Activation cluster ROIs were defined in the PPA bilaterally at $p<.05$, familywise error (FWE) corrected for the whole brain mass using the group level contrast between building target definition and face target definition. The reverse contrast (face target definition minus building target definition) failed to reliably identify a region corresponding to the FFA (see results below). The data from the PPA regions were extracted using MARSBAR and analyzed using SPSS.

\section{Tasks 3 and 4: Response Versus No Response \\ at Target Detection}

Fourteen participants undertook a further two versions of the target detection task. The two modified versions of the task were designed to control for any possible requirement for response suppression while identifying frontoparietal subregions preferentially recruited during generation of an overt motor response. As before, participants monitored sequences of stimuli for the presentation of a target object defined at the start of the sequence. In Task 3, positive or negative responses were made when probed at the end of the sequence in the same way as for Tasks 1 and 2. In Task 4, there was no probe; instead, an immediate buttonpress was made whenever a target was detected. Participants monitored 32 sequences of nonoverlapping faces and buildings for the presentation of the target object in each version of the task. The two versions of the task used independent stimulus sets, each of which was composed of four faces and four buildings. When a face was sought, only faces were presented, whereas only buildings were presented when a building was sought. All stimuli were presented at equal frequency; as a consequence, one in four monitored stimuli depicted the current target object. Participants monitored a total of 32 targets and 96 distractors from the same category as the target in each version of the task.

Scanning was carried out at the MRC Cognition and Brain Sciences Unit using a 3 Tesla Siemens Trim Trio scanner. Thirty-two 3-mm slices (1-mm interslice gap) were acquired using a TR of $2 \mathrm{sec}$ 


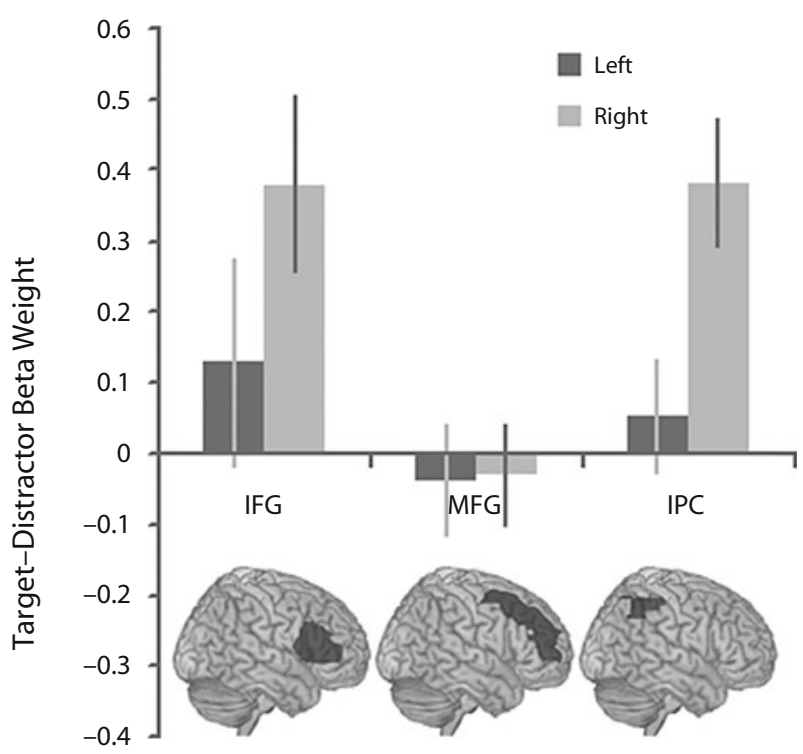

Figure 2. The BOLD response to infrequent targets. Task 1: Difference in the BOLD response to targets and distractors in the frontoparietal ROIs. The BOLD response was significantly greater to targets than to distractors in the right IFG and the right IPC.

and in-plane resolution of $3 \times 3 \mathrm{~mm}$. A total of $235 \mathrm{~T} 2$-weighted echo-planar images depicting BOLD contrast were acquired in each task, with the first 10 discarded to avoid T1 equilibrium effects.

Images were preprocessed and analyzed using SPM5 (Wellcome Department of Cognitive Neurology). Prior to analysis, images were slice time corrected, reoriented to correct for subject motion, spatially normalized to the standard Montreal Neurological Institute template, and smoothed with an 8-mm full-width at half-maximum Gaussian kernel. Data were also high-pass filtered prior to analysis (cutoff period $128 \mathrm{sec}$ ). Fixed effects analyses were carried out on each participant's data, using general linear models. Regressor functions were created by convolving timing functions indicating the onset and duration of each event, with a basis function representing the canonical hemodynamic response. Each regressor function was then entered into the model as a predictor variable, seven of which were entered into the linear model; they represented face target definition, building target definition, face target presentations, face distractor presentations, building target presentations, building distractor presentations, and the probe stimulus with response.

Group level analyses were carried out using the same six ROIs as in the first two versions of the task. For each participant, responses to target and distractor stimuli estimated by the fixed effects analyses were averaged across voxels within each of these ROIs, using the MARSBAR toolbox (Brett et al., 2002). These mean values were then exported for analysis using SPSS.

\section{RESULTS}

\section{Target-Sensitive Neural Response in the Frontal and Parietal Cortex}

Data from the first task, in which targets were less frequent than distractors, and the second task, in which targets and distractors occurred with equal frequency, were extracted from the IFG, MFG, and IPC ROIs for the contrast of target minus distractor presentation (Figures 2 and 3). The data from the frontal cortex ROIs indicated an increase in the BOLD response during target presen- tation selectively in the right IFG during both versions of the task. The significance of this result was examined using a three-way repeated measures ANOVA with target frequency (Task 1 vs. Task 2), ROI (IFG vs. MFG), and hemisphere (left vs. right) as factors. A significant main effect of ROI was evident $[F(1,13)=9.77, p<.01]$, with a significant ROI $\times$ hemisphere interaction $[F(1,13)=$ $5.75, p<.05]$ favoring heightened BOLD response to targets in the right IFG. There were no significant interactions with, or main effects of, target frequency. These results indicate that the target selective BOLD response in the right IFG is not dependent on the relative infrequency of target presentation. One-sample $t$ tests against 0 (onetailed) confirmed this result with significant increases in the BOLD response to targets in the right IFG in Task 1 and in the IFG bilaterally in Task 2 (Task 1 left IFG $t=$ $0.89, p=.19$, right IFG $t=3.09, p<.005$; Task 2 left IFG $t=1.92, p<.05$, right IFG $t=1.79, p<.05)$. Note that the MFG ROIs are more extensive than the IFG ROIs. To rule out the possibility that a subportion of the right MFG was responding selectively to targets, we divided the right MFG ROI into a posterior and an anterior portion at $y=$ 30. Examination of target versus distractor contrast collapsed across the first two versions of the task revealed no significant increase in the BOLD response in either the posterior or anterior portion of the MFG (posterior MFG $t=-0.04, p=.52$, anterior MFG $t=-0.03, p=.511)$.

A greater BOLD response to targets in the right but not the left IPC was also apparent. The significance of this result was examined using a two-way ANOVA in which the factors were target frequency (Task 1 vs. Task 2) and hemisphere (left vs. right). A significant main effect of hemisphere was observed $[F(1,13)=8.63, p=.01]$, with

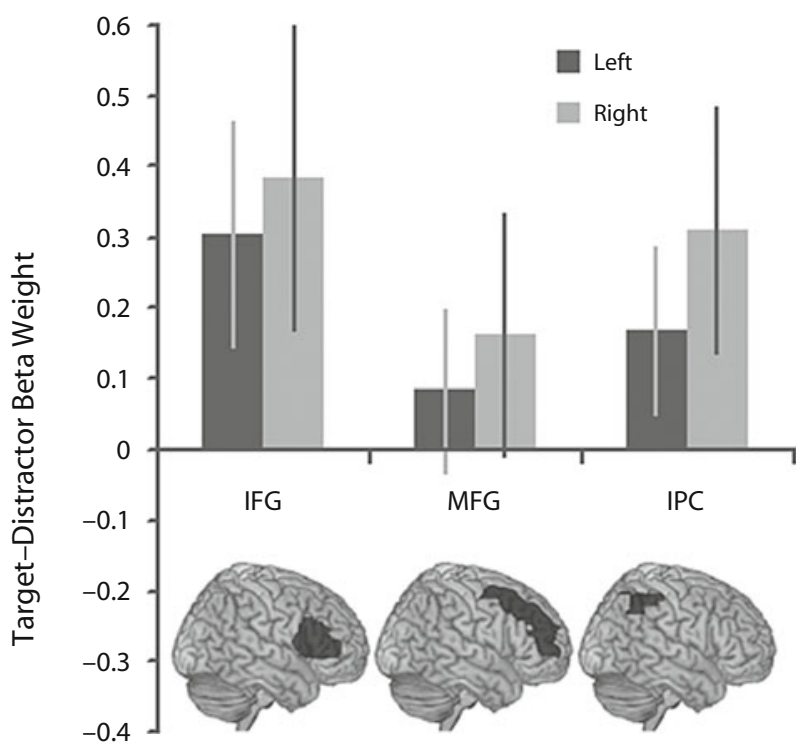

Figure 3. The BOLD response to frequent targets. Task 2: The BOLD response was greater to targets than to distractors in the IFG bilaterally even when the targets were presented in $50 \%$ of the trials. The IPC followed a similar subthreshold trend. 

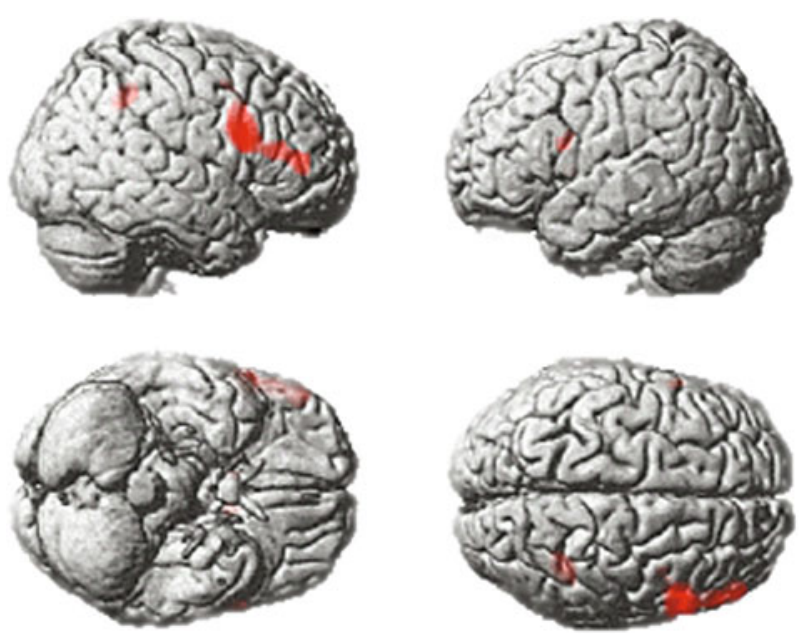

Figure 4. Whole brain analysis. A whole brain analysis collapsed across Tasks 1 and 2 presented at $p<.05$, FDR corrected for the whole brain mass. Significant activation foci were apparent in the IFG bilaterally and the right IPC (see Table 1).

no significant effect of frequency, and no interaction. One-sample $t$ tests against 0 (one-tailed) confirmed this result, with significant activation in the right IPC in both versions of the task (Task 1 left IPC $t=0.66, p=.26$, right IPC $t=4.21, p<.001$; Task 2 left IPC $t=1.42, p=$ .07 , right IPC $t=1.76, p=.05$ ).

The whole brain maps depicting beta weightings for the target and distractor regressors were also examined in a $2 \times 2$ full factorial model in SPM5 in which the factors were target frequency (Task 1 vs. Task 2) and relevance (target vs. distractor). Examination of the statistical parametric maps (Figure 4) ( $p<.05$, FDR corrected) confirmed the main result from the ROI analyses, with peak activation foci (Table 1) observed in the right inferior frontal gyrus during the presentation of targets versus distractors. In addition, activation foci were observed in the left IFG, regions of the striatum, the middle temporal gyrus, and the IPC for this contrast (Table 1). In line with the results from the ROI analyses, there were no significant voxels for the interaction between frequency and relevance, even when the whole brain statistical maps were examined at a liberal threshold of $p<.005$, uncorrected.

Data from the third task, in which a buttonpress was made when probed at the end of the sequence, and the fourth task, in which a buttonpress was made at the point of target detection, were extracted from the IFG, MFG, and IPC ROIs for target minus distractor presentation. Data from the frontal ROIs indicated a particularly high BOLD response to targets compared with distractors in the right IFG in both versions of the task (Figures 5A and 5B). This result was examined using a three-way ANOVA in which the factors were response (Task 3 vs. Task 4), ROI (IFG vs. MFG), and hemisphere (left vs. right). There was a significant interaction of response $\times$ ROI $\times$ hemisphere $[F(1,13)=$ $13.26, p<.005]$, an interaction of response $\times$ hemisphere $[F(1,13)=20.69, p<.001]$, as well as a main effect of hemisphere $[F(1,13)=32.15, p<.001]$. To clarify the results, data for each were examined further in two separate repeated measures ANOVAs, each with the factors ROI and hemisphere. The Task 3 ANOVA revealed a significant main effect of hemisphere $[F(1,13)=11.66, p=.005]$ and a significant main effect of ROI $[F(1,13)=4.72, p<.05]$ favoring the right IFG, with no significant interaction. The results from Task 4 revealed a significant main effect of hemisphere $[F(1,13)=17.24, p=.001]$ and a significant interaction of ROI $\times$ hemisphere $[F(1,13)=10.90, p<$ $.01]$ favoring the right IFG, with no significant main effect of ROI. One-sample $t$ tests against 0 (one-tailed) confirmed these results with significantly greater BOLD response to targets compared with distractors in the right IFG in both versions of the task (Task 3 left IFG $t=0.25, p=.40$, right IFG $t=2.99, p=.005$; Task 4 left IFG $t=0.57, p=.29$, right IFG $t=4.51, p<.001$ ), and a smaller effect in the right MFG (Task 3 left MFG $t=-1.10, p=.85$, right MFG $t=1.91, p<.05$; Task 4 left MFG $t=1.24, p=.12$, right MFG $t=1.8, p<.05)$.

Data extracted from the IPC ROIs for the third and fourth task showed an increased BOLD response to targets, particularly when an overt motor response was made at the point of target detection (Figure 6). This result was examined in a $2 \times 2$ ANOVA in which the conditions were response (Task 3 vs. Task 4 ) and hemisphere (left vs. right). The results revealed a subthreshold trend toward increased activation in the response condition $[F(1,13)=$ $3.89, p=.07]$, with no main effect of hemisphere and no interaction. One-sample $t$ tests against 0 (one-tailed) confirmed this result (Task 1 left IPC $t=1.19, p=.13$, right IPC $t=2.52, p<.01$; Task 2 left IPC $t=4.11, p<.001$, right IPC $t=3.84, p=.001$ ).

The whole brain maps depicting the beta weights for target and distractor regressors were also examined using a full factorial model in SPM5. The factors were response (Task 3 vs. Task 4) and relevance (target vs. distractor). In line with the results from the ROI analyses, there was a significant (FDR corrected for the whole brain mass at $p<.05$ ) main effect of relevance favoring heightened BOLD response to targets in a predominantly right-lateralized network of brain regions including the right IFG, and sensory motor cortex spreading up to the right IPC and the right middle temporal gyrus (Figure 7A and Table 2A). The response $\times$ relevance

Table 1

Whole Brain Analysis From Tasks 1 and 2

\begin{tabular}{lcrrrrc}
\hline & Approx. BA & \multicolumn{1}{c}{$x$} & \multicolumn{1}{c}{$y$} & \multicolumn{1}{c}{$z$} & \multicolumn{1}{c}{$T$} & $p$ (FDR) \\
\hline Left inferior frontal gyrus & 45 & -58 & 10 & 20 & 4.08 & .029 \\
Right inferior frontal gyrus & 45 & 56 & 12 & 24 & 4.48 & .028 \\
Right inferior frontal gyrus & 46 & 50 & 42 & 8 & 3.92 & .033 \\
Right inferior parietal lobule & 40 & 40 & -48 & 44 & 4.62 & .028 \\
\hline
\end{tabular}


A

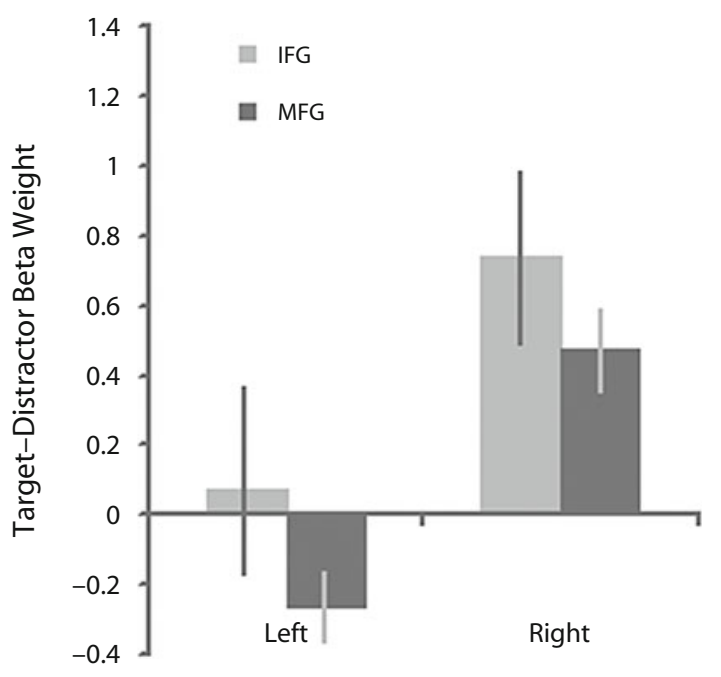

B

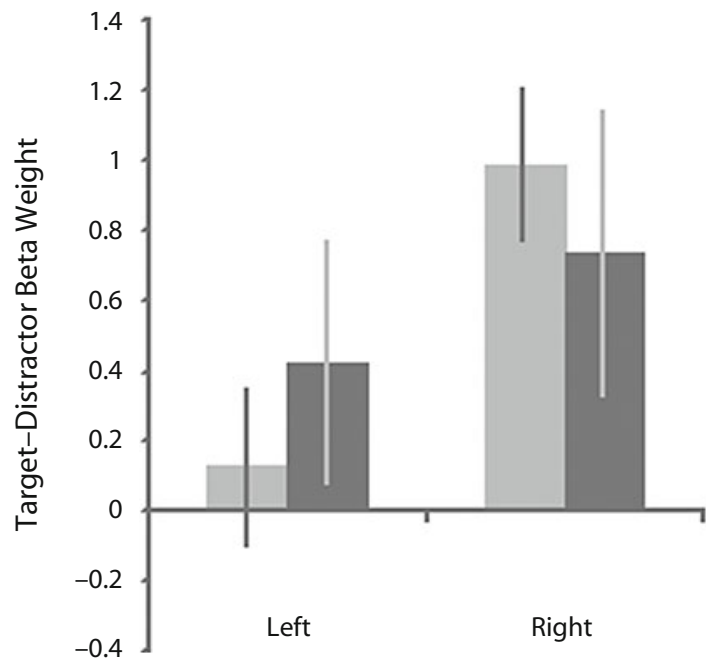

Figure 5. The effect of immediate motor response to targets in the frontal ROIs. (A) BOLD response to targets in the frontal ROIs in Task 3, where overt motor responses were made when probed at the end of the sequence. (B) BOLD response to targets in Task 4, where a motor response is made at the point of target detection.

interaction also rendered a network of brain regions with heightened BOLD response to targets when an overt motor response was made at the point of target detection. These included clusters in the sensory motor cortex spreading up to the IPC bilaterally, the anterior cingulate bilaterally, and regions within the cerebellum (Figure $7 \mathrm{~B}$ and Table $2 \mathrm{~B}$ ). The results rule out any possibility that the right IFG response to targets is related specifically to the suppression of an overt motor response, and implicate a further network of brain regions, in response generation.

\section{Attentional Modulation of the Neural Response in Category-Sensitive Brain Regions}

To define category-specific processing area regions for Tasks 1 and 2 (see the Method section), we used data from the stage at which new targets were defined (when a face or a building alone was on the screen). A contrast subtracting responses evoked by face targets from responses to building targets showed that the latter were associated with increased BOLD response bilaterally in the parahippocampus in two discrete clusters at MNI coordinates $-28,-46,-14$ in the left hemisphere, and 26, -42, -16 in the right hemisphere. These coordinates survived FWE correction for the whole brain mass at $p<.05$, and are close to those expected for the PPA (Epstein \& Kanwisher, 1998). These clusters were used as ROIs for further analysis (Figure 8A). The reverse contrast did not reveal significant activation near the expected coordinates for the FFA (Kanwisher et al., 1997) even at a low threshold of $p<$ .05 , uncorrected. Due to the small size of this region, coordinates are often defined on an individual subject level; even at this level, however, significant FFA activation was not observed. The use of focal ROIs at the average coordinates reported by Kanwisher et al. (1997) also showed no significant increases in activity during trials where a face was the target stimulus.
Responses to target and distractor stimuli were extracted from the PPA ROIs (Figure 8B) and were examined in SPSS using a repeated measures ANOVA in which the factors were frequency (infrequent vs. frequent), attended category (faces or buildings), relevance (target or distractor), and hemisphere. There was a significant main effect of attended category $[F(1,13)=31.41, p<.001]$, with greater activation during sustained attention to buildings, modulated by a significant interaction of hemisphere $X$ category $[F(1,13)=9.37, p<.01]$.

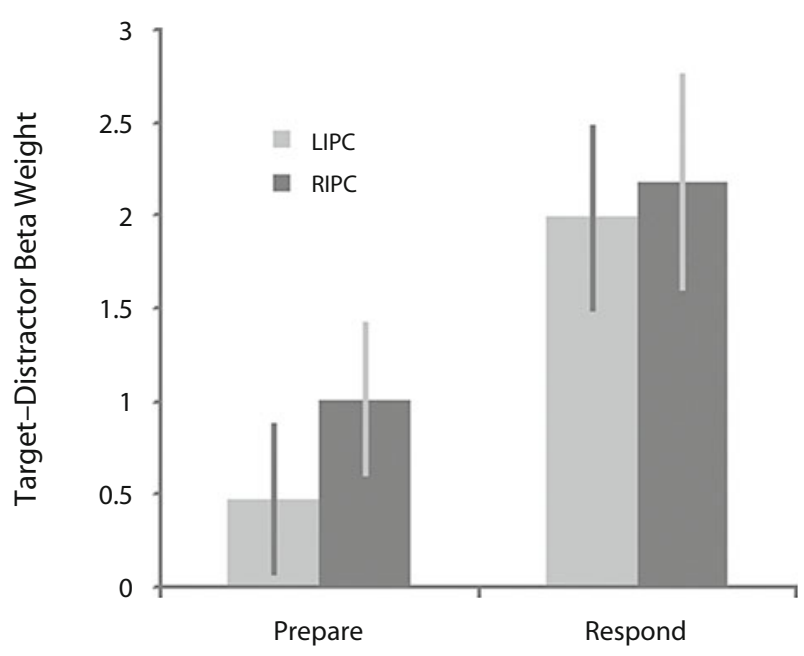

Figure 6. Increased BOLD response in the IPC during motor response to targets. The IPC ROIs showed a subthreshold trend toward increased BOLD response to targets when a buttonpress was made at the point of target detection in Task 4 (Respond), compared with when the buttonpress was made when probed at the end of the sequence in Task 3 (Prepare). 

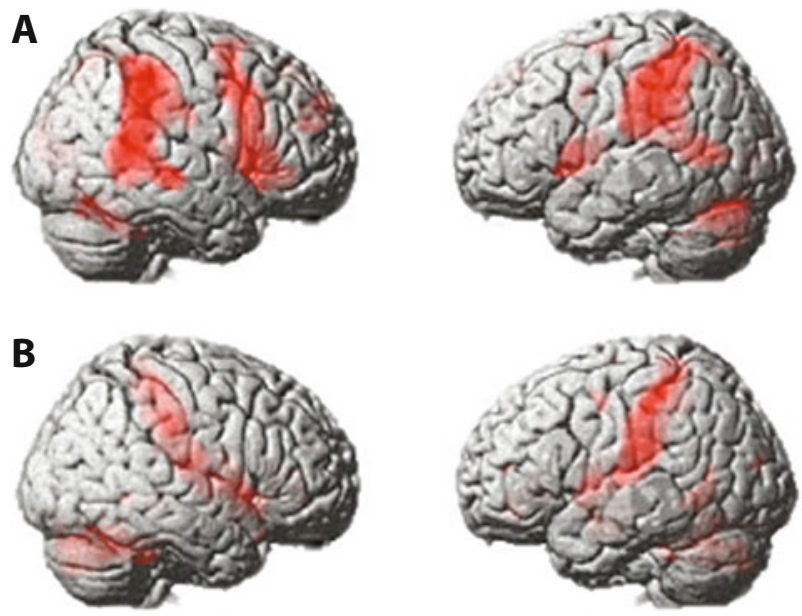

Figure 7. Main effect of target detection and interaction with motor response. (A) Main effect of target versus distractor from the full factorial analysis of Tasks 3 and 4 presented at $p<.05$, FDR corrected for the whole brain mass. A dominantly right lateralized network was evident, including IFG, IPC, and temporal cortex. (B) Interaction of Task 3 versus Task 4 with the presentation of targets versus distractors. Increased BOLD response to targets was apparent in a network of brain regions when an overt motor response was generated at the point of target detection (Task 4). Activation clusters spread across sensory motor cortex extending into the IPC. Bilateral anterior cingulate cortex and bilateral regions of the cerebellum were also more activated in this contrast.

\section{DISCUSSION}

Our results clearly demonstrate that the right IFG, in particular, rapidly tunes to selectively respond to current targets, and becomes less responsive to those same objects when the task demands change in several different task contexts. In line with models that posit a global adaptive system for working memory and attention (Dehaene, Kerszberg, \& Changeux, 1998; Duncan, 2001; Miller \& Cohen, 2001; Norman \& Shallice, 1986), this ability appears to be generalized across different stimulus categories.

Previously, using a similar task design, we reported significant differences in the extent of selective tuning in different components of the frontoparietal network (Hampshire et al., 2007). While monitoring nonoverlapping stimuli for the presentation of a target object, we observed the IFG to respond tightly to the current target only, but the MFG to respond also to distractor objects drawn from the same category as that target. The results presented here again reveal a significant difference between the profile of the BOLD response in the IFG and the MFG, with the MFG showing little or no significant activation differences between the presentation of the target or a distractor object from the same category as the target.

A number of functional specializations have previously been proposed between subregions of the frontoparietal network (Corbetta \& Shulman, 2002; D'Esposito et al., 1999; Koechlin, Basso, Pietrini, Panzer, \& Grafman, 1999; Owen, 1997; Petrides, 1994, 1995, 2005; Ramnani $\&$ Owen, 2004). However, the precise nature of these specializations remains controversial. The current uncertainty regarding the nature of frontoparietal organization is, to a large extent, driven by the use of complex, cognitively heterogeneous tasks that confound multiple cognitive factors when one attempts to functionally dissociate frontoparietal subregions. For example, reversing a string of digits maintained in working memory (Owen, Lee, \& Williams, 2000) involves multiple components apart from simple manipulation, including the maintenance and sequencing of a strategy, rehearsal, and general difficulty level. Our finding of an IFG/MFG dissociation within the context

Table 2

Whole Brain Analysis From Tasks 3 and 4

\begin{tabular}{lrrrrrr}
\hline & Approx. BA & $x$ & $y$ & $z$ & $T$ & $p(\mathrm{FDR})$ \\
\hline \multicolumn{7}{c}{ Target-Distractor } \\
Left inferior frontal gyrus & 44 & -46 & 0 & 10 & 6.51 & $<.001$ \\
Right inferior frontal gyrus & 9 & 60 & 18 & 26 & 7.36 & $<.001$ \\
Right inferior frontal gyrus & 45 & 56 & 16 & 6 & 6.79 & $<.001$ \\
Right medial frontal gyrus & 8 & 6 & 44 & 46 & 5.7 & $<.001$ \\
Right superior frontal gyrus & 11 & 20 & 50 & -16 & 4.02 & $<.005$ \\
Left inferior parietal lobule & 40 & -44 & -46 & 56 & 7.53 & $<.001$ \\
Right inferior parietal lobule & 40 & 50 & -36 & 50 & 6.9 & $<.001$ \\
Right cingulate gyrus & 23 & 10 & -20 & 32 & 3.34 & $<.01$ \\
Right insula & 13 & 34 & 20 & 6 & 3.31 & $<.01$ \\
Right middle temporal gyrus & 21 & 60 & -48 & 8 & 8.17 & $<.001$ \\
Right superior temporal gyrus & 38 & 52 & 10 & -26 & 3.77 & $<.005$
\end{tabular}

Interaction of Response (Task 3 vs. Task 4) $\times$ Relevance (i.e., Increased Activation to Target With Immediate Response)

Left inferior parietal lobule

Right inferior parietal lobule

Left anterior cingulate

Right anterior cingulate

Left insula

Right transverse temporal gyrus

Left cerebellum

Right cerebellum

Right amygdala

$\begin{array}{rrrrll}40 & -54 & -38 & 54 & 6.36 & <.001 \\ 40 & 54 & -32 & 56 & 5.16 & <.001 \\ & -18 & 34 & 4 & 4.9 & =.001 \\ & 18 & 34 & 0 & 5.68 & <.001 \\ 13 & -40 & -4 & 10 & 5.91 & <.001 \\ 42 & 66 & -10 & 14 & 5.19 & <.001 \\ & -24 & -46 & -26 & 4.95 & =.001 \\ & 24 & -46 & -28 & 8.24 & <.001 \\ & 20 & -4 & -16 & 4.65 & =.001\end{array}$


A
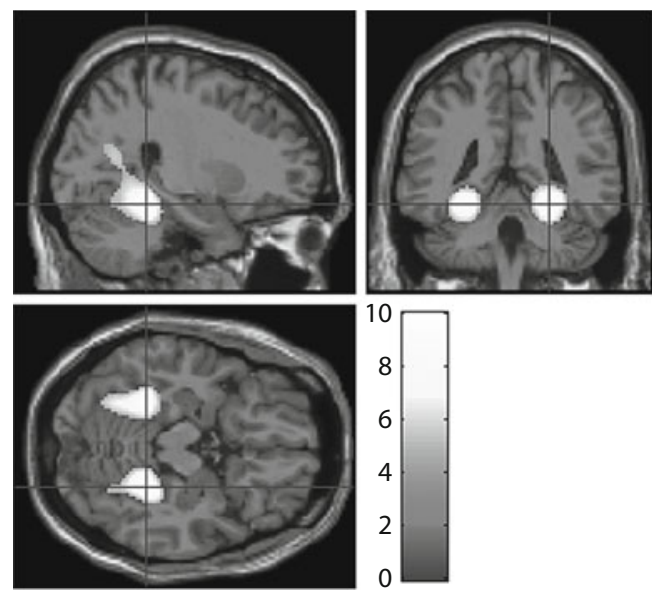

B

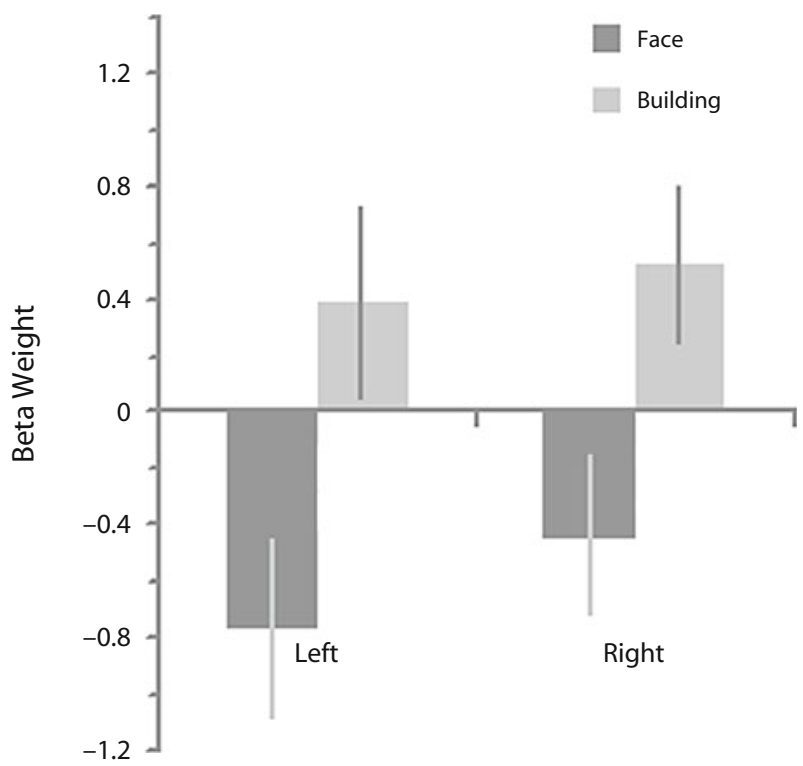

Figure 8. Attentional modulation in the parahippocampal gyrus. (A) Whole brain activation when building targets were defined minus when face targets were defined (when only a face or a building was presented on the screen). (B) Data extracted from the monitored sequences of targets and distractors (when a face and building were always present in the compound stimulus pairs). The PPA was more active bilaterally when monitoring the building components compared with the face components of the compound stimuli.

of a simple target detection task affords an important new insight into the nature of frontoparietal organization. On the basis of these results and those from the broader literature, we propose that the representation within the right IFG rapidly adapts in order to respond to those items at the current focus of intended action (Hampshire, Thompson, Duncan, \& Owen, 2008). The IFG lies at the crossover point between bottom-up, stimulus-driven processing and the processing of top-down goal-oriented intentions, and therefore responds particularly strongly when the currently attended item is at the focus of an individual's current intentions. In contrast, the anatomically adjacent MFG may play a more abstract role in planning and monitoring goal-oriented behavior (Petrides, 1994, 1995, 2005). This account accords well with the previous suggestion that the IFG is involved in first-order executive tasks, such as actively maintaining items in working memory, whereas the MFG is involved in higher order executive tasks, such as planning, structuring, monitoring, and manipulating working memory items (D’Esposito et al., 1999; Murray \& Ranganath, 2007; Owen et al., 2000; Petrides, 1994, $1995,2005)$. Selective response to targets is also broadly consistent with the proposition that the IFG forms part of a ventral circuit for attentional orienting, responding to items of particular relevance to immediate behavior (Corbetta \& Shulman, 2002). However, the results of this study extend those previous results by demonstrating that the IFG tunes rapidly on a stimulus-by-stimulus basis, according to whatever aspect of the environment is the current focus of attention. Furthermore, the results from the frequency control condition demonstrate that this target-selective tuning occurs even when targets occur at a $50 \%$ frequency, and are therefore just as expected as the distractor objects. This result rules out surprise or expectancy violation as a possible causal factor in the current task design.

Target detection paradigms classically use an imbalance in the ratio between targets and distractors to ensure that participants do not reconfigure their intended task schemas from the detection of specific targets to sorting between two stimuli or stimulus categories of equal relevance to task performance (Linden et al., 1999). Having a response that depends only on the target information - for example, target naming in a visual search task-is an alternative technique that works to a similar end. In either case, there are potential problems for interpretation of the results. In the former case, the infrequency of the target stimulus or of the planned positive response could potentially account for observed activation differences. Likewise, in the latter case, target-related activation could be attributed to the additional actions undertaken on detection. We sought to examine target detection free from these confounds in the second version of our task by presenting targets and distractors with a 1:1 frequency. Somewhat surprisingly, the target selective BOLD response in the IFG was still evident to the same extent. This finding adds considerable weight to the proposition that the representation within the IFG rapidly tunes in order to respond selectively to those items that form the basis of the current task schema.

Although the present study has focused on functional specialization within the frontal cortex, it is important to note that the right IPC was also significantly activated during the presentation of target objects. The functions of the IPC are generally hard to dissociate from those of the lateral prefrontal cortex in neuroimaging experiments (Duncan, 2006), and the right IPC presents a similar profile to the IFG's. The trend toward increased response to targets when an overt motor response was made at the point of detection would suggest that, in line with findings from the electrophysiology literature (Cohen \& Andersen, 
2002), the IPC is involved in mapping between stimuli and responses. However, it is important not to rule out the possibility that the IPC also plays a more central role in executive tasks.

It has previously been proposed that the attentional system exerts its effects by modulating competition for resources in less adaptable category-specific processing streams (Desimone \& Duncan, 1995). One prediction from this hypothesis is that brain regions sensitive to the presentation of stimuli from particular categories - for example, the PPA (Epstein \& Kanwisher, 1998) or the FFA (Kanwisher et al., 1997) — should be differentially affected not only by the presentation of stimuli drawn from those categories (buildings and faces, respectively), but also by the sustained focusing of attention on those categories of information (O'Craven, Downing, \& Kanwisher, 1999). Here, we tested this hypothesis by contrasting sequences where the face or building aspects of the compound stimuli were being monitored. During sustained attention to buildings, increased activation was observed bilaterally in the PPA ROIs.

An important aside is the relevance of the current findings to those studies of attention that have implicated the right IFG in response suppression, most typically in the currently popular go/no-go tasks (Aron et al., 2003a, 2003b; Rubia et al., 2003). In go/no-go tasks, participants respond to the regular presentation of a go signal, and must inhibit that routine response when an infrequent stop signal is subsequently presented. It seems plausible that a large component in this type of task is the detection of the stop signal, a task demand very similar to the detection of a learned target stimulus. The resultant output of the detection of the stop signal is the planned and intended suppression of the recently initiated motor response. This output is most probably facilitated by the top-down biasing signals considered to be one of the key mechanisms by which the frontoparietal network exerts executive control over other brain systems (Desimone \& Duncan, 1995). It is unsurprising, therefore, that a similar network is activated when inhibiting a routine response, as well as when detecting target objects. In our present study, the same activity was observed in the right IFG both when targets were less frequent or equally frequent to distractors, and when an overt response was withheld until probed, or made at the point of target detection. In light of this finding, it seems reasonable to suggest that right IFG activity at the point of inhibition in the go/no-go task could be just another example of target detection with planned response; and, although deficits on this task could be due to an inability to inhibit dominant responses, they could also be due to more general difficulties in maintaining the focus of attention. In that respect, it is important to separate inhibitory control in go/no-go tasks, where a planned action is undertaken, from inhibitory control in the more classical sense, where the plan is changed in response to suboptimal feedback from the environment. It is well established that inhibitory control in the more classical sense is also dependent on other components of the executive system, including the orbitofrontal cortices
(Dias, Robbins, \& Roberts, 1996; Hampshire \& Owen, 2006; Hornak et al., 2004).

In summary, the findings presented here accord with a special role for the right IFG in selectively tuning to respond to those objects that are at the current focus of intention. This region forms a component of the wider attentional network, which exerts its effects by modulating processing in less adaptable category-sensitive brain regions.

\section{AUTHOR NOTE}

This research was supported by Medical Research Council Grant U1055.01.002.00001.01. The authors thank the study participants and the radiographers. Correspondence concerning this article should be addressed to A. Hampshire, MRC Cognition and Brain Sciences Clinic, 15 Chaucer Road, Cambridge CB2 7EF, England (e-mail: adam .hampshire@mrc-cbu.cam.ac.uk).

\section{REFERENCES}

Aron, A. R., Fletcher, P. C., Bullmore, E. T., Sahakian, B. J., \& Robisns, T. W. (2003a). Stop-signal inhibition disrupted by damage to right inferior frontal gyrus in humans. Nature Neuroscience, $\mathbf{6}$, 115-116.

Aron, A. R., Fletcher, P. C., Bullmore, E. T., Sahakian, B. J., \& RobBins, T. W. (2003b). Stop-signal inhibition disrupted by damage to right inferior frontal gyrus in humans: Erratum. Nature Neuroscience, 6, 1329.

Bor, D., Duncan, J., \& Owen, A. M. (2001). The role of spatial configuration in tests of working memory explored with functional neuroimaging. Scandinavian Journal of Psychology, 42, 217-224.

Braver, T. S., Reynolds, J. R., \& Donaldson, D. I. (2003). Neural mechanisms of transient and sustained cognitive control during task switching. Neuron, 39, 713-726.

Brett, M., Anton, J., Valabregue, R., \& Poline, J. (2002, June). Region of interest analysis using an SPM toolbox. Paper presented at the 8th International Conference on Functional Mapping of the Human Brain, Sendai, Japan.

Cohen, Y. E., \& Andersen, R. A. (2002). A common reference frame for movement plans in the posterior parietal cortex. Nature Reviews Neuroscience, 3, 553-562.

Corbetta, M., \& Shulman, G. L. (2002). Control of goal-directed and stimulus-driven attention in the brain. Nature Reviews Neuroscience, 3, 201-215.

Cusack, R., Brett, M., \& Osswald, K. (2003). An evaluation of the use of magnetic field maps to undistort echo-planar images. NeuroImage, 18, 127-142.

Dehaene, S., Kerszberg, M., \& Changeux, J. P. (1998). A neuronal model of a global workspace in effortful cognitive tasks. Proceedings of the National Academy of Sciences, 95, 14529-14534.

Desimone, R., \& Duncan, J. (1995). Neural mechanisms of selective visual attention. Annual Review of Neuroscience, 18, 193-222.

D’Esposito, M., Postle, B. R., Ballard, D., \& Lease, J. (1999). Maintenance versus manipulation of information held in working memory: An event-related fMRI study. Brain Cognition, 41, 66-86.

Dias, R., Robbins, T. W., \& RoberTs, A. C. (1996). Dissociation in prefrontal cortex of affective and attentional shifts. Nature, 380, 69-72.

Dove, A., Pollmann, S., Schubert, T., Wiggins, C. J., \& von CraMON, D. Y. (2000). Prefrontal cortex activation in task switching: An event-related fMRI study. Brain Research, 9, 103-109.

Dreher, J. C., Koechlin, E., Ali, S. O., \& Grafman, J. (2002). The roles of timing and task order during task switching. NeuroImage, 17, 95-109.

Duncan, J. (2001). An adaptive coding model of neural function in prefrontal cortex. Nature Reviews Neuroscience, 2, 820-829.

DUNCAN, J. (2006). EPS mid-career award 2004: Brain mechanisms of attention. Quarterly Journal of Experimental Psychology, 59, 2-27.

Duncan, J., \& OWEN, A. M. (2000). Common regions of the human frontal lobe recruited by diverse cognitive demands. Trends in Neurosciences, 23, 475-483. 
Epstein, R., \& Kanwisher, N. (1998). A cortical representation of the local visual environment. Nature, 392, 598-601.

Everling, S., Tinsley, C. J., Gaffan, D., \& Duncan, J. (2002). Filtering of neural signals by focused attention in the monkey prefrontal cortex. Nature Neuroscience, 5, 671-676.

Fletcher, P. C., \& Henson, R. N. (2001). Frontal lobes and human memory: Insights from functional neuroimaging. Brain, 124, 849-881.

Freedman, D. J., Riesenhuber, M., Poggio, T., \& Miller, E. K. (2001). Categorical representation of visual stimuli in the primate prefrontal cortex. Science, 291, 312-316.

Hampshire, A., Duncan, J., \& Owen, A. M. (2007). Selective tuning of the blood oxygenation level-dependent response during simple target detection dissociates human frontoparietal subregions. Journal of Neuroscience, 27, 6219-6223.

Hampshire, A., \& Owen, A. M. (2006). Fractionating attentional control using event-related fMRI. Cerebral Cortex, 16, 1679-1689.

Hampshire, A., Thompson, R., Duncan, J., \& Owen, A. M. (2008). The target selective neural response-Similarity, ambiguity, and learning effects. PLOS ONE, 3, e2520.

Hornak, J., O’Doherty, J., Bramham, J., Rolls, E. T., Morris, R. G., Bullock, P. R., \& Polkey, C. E. (2004). Reward-related reversal learning after surgical excisions in orbito-frontal or dorsolateral prefrontal cortex in humans. Journal of Cognitive Neuroscience, 16, 463-478.

Kanwisher, N., McDermott, J., \& Chun, M. M. (1997). The fusiform face area: A module in human extrastriate cortex specialized for face perception. Journal of Neuroscience, 17, 4302-4311.

Kimberg, D. Y., Aguirre, G. K., \& D'Esposito, M. (2000). Modulation of task-related neural activity in task-switching: An fMRI study. Cognitive Brain Research, 10, 189-196.

Koechlin, E., Basso, G., Pietrini, P., Panzer, S., \& Grafman, J. (1999). The role of the anterior prefrontal cortex in human cognition. Nature, 399, 148-151.

Linden, D. E. J., Prvulovic, D., Formisano, E., Vollinger, M., Zanella, F. E., Goebel, R., \& Dierks, T. (1999). The functional neuroanatomy of target detection: An fMRI study of visual and auditory oddball tasks. Cerebral Cortex, 9, 815-823.

Miller, E. K., \& CoHen, J. D. (2001). An integrative theory of prefrontal cortex function. Annual Review of Neuroscience, 24, 167-202.

Murray, L. J., \& Ranganath, C. (2007). The dorsolateral prefrontal cortex contributes to successful relational memory encoding. Journal of Neuroscience, 27, 5515-5522.

Norman, D. A., \& Shallice, T. (1986). Attention to action: Willed and automatic control of behavior. In R. Davidson, G. E. Schwartz, \& D. Shapiro (Eds.), Consciousness and self-regulation: Advances in research and theory (Vol. 4, pp. 1-18). New York: Plenum.

O'Craven, K. M., Downing, P. E., \& Kanwisher, N. (1999). fMRI evidence for objects as the units of attentional selection. Nature, 401, 584-587.
Owen, A. M. (1997). The functional organization of working memory processes within human lateral frontal cortex: The contribution of functional neuroimaging. European Journal of Neuroscience, 9, 1329-1339.

Owen, A. M., \& Hampshire, A. (in press). The mid-ventrolateral frontal cortex and attentional control. In F. Roesler \& C. Ranganath (Eds.), Neuroimaging and psychological theories of human memory. Oxford: Oxford University Press.

Owen, A. M., Lee, A. C. H., \& Williams, E. J. (2000). Dissociating aspects of verbal working memory within the human frontal lobe: Further evidence for a process-specific model of lateral frontal organization. Psychobiology, 28, 146-155.

Peelen, M. V., Heslenfeld, D. J., \& Theeuwes, J. (2004). Endogenous and exogenous attention shifts are mediated by the same largescale neural network. NeuroImage, 22, 822-830.

Petrides, M. (1994). Frontal lobes and working memory: Evidence from investigations of the effects of cortical excisions in nonhuman primates. In F. Boller \& J. Grafman (Eds.), Handbook of neuropsychology (Vol. 9, pp. 59-82). Amsterdam: Elsevier.

Petrides, M. (1995). Impairments on nonspatial self-ordered and externally ordered working-memory tasks after lesions of the mid-dorsal part of the lateral frontal cortex in the monkey. Journal of Neuroscience, 15, 359-375.

Petrides, M. (2005). Lateral prefrontal cortex: Architectonic and functional organization. Philosophical Transactions of the Royal Society $B, \mathbf{3 6 0}, 781-795$.

Postle, B. R., \& D'Esposito, M. (2000). Evaluating models of the topographical organization of working memory function in frontal cortex with event-related fMRI. Psychobiology, 28, 132-145.

RAMNANI, N., \& OWEN, A. M. (2004). Anterior prefrontal cortex: Insights into function from anatomy and neuroimaging. Nature Reviews Neuroscience, 5, 184-194.

Rubia, K., Smith, A. B., Brammer, M. J., \& Taylor, E. (2003). Right inferior prefrontal cortex mediates response inhibition while mesial prefrontal cortex is responsible for error detection. NeuroImage, $\mathbf{2 0}$, 351-358.

Sohn, M.-H., Ursu, S., Anderson, J. R., Stenger, V. A., \& Carter, C. S. (2000). The role of prefrontal cortex and posterior parietal cortex in task switching. Proceedings of the National Academy of Sciences, 97, 13448-13453.

Tzourio-Mazoyer, N., Landeau, B., Papathanassiou, D., Crivello, F., Etard, O., Delcroix, N., ET Al. (2002). Automated anatomical labeling of activations in SPM using a macroscopic anatomical parcellation of the MNI MRI single-subject brain. NeuroImage, 15, 273-289.

(Manuscript received April 23, 2008; revision accepted for publication August 11, 2008.) 